\title{
Reducing Residual Moveout for Long Offset Data in VTI Media Using Padé Approximation
}

\author{
Indah Nur Pratiwi ${ }^{1}$, Mohammad Syamsu Rosid ${ }^{1, *}$, and Humbang Purba ${ }^{2}$ \\ ${ }^{1}$ Departement of Physics, Faculty of Mathematics and Natural Science, University of Indonesia, Depok-Indonesia \\ 2 PPPTMBG LEMIGAS, Jakarta-Indonesia
}

\begin{abstract}
Modification of the hyperbolic travel time equation into non-hyperbolic travel time equation is important to increase the reduction residual moveout for long offset data. Some researchers have modified hyperbolic travel time equation into a non-hyperbolic travel time equation to obtain a more accurate value NMO velocity and parameter an-ellipticity or etha on the large offset to depth ratio (ODR) so that the residual moveout value is smaller mainly in large offset to depth ratio. The aims of research is to increase the reduction value of error residue at long offset data using Padé approximation then compare with several approximations. The method used in this study is to conduct forward modeling of the subsurface coating structure. The results of the three-dimensional analysis show that the Padé approximation has the best accuracy compared to the other travel time equations for ODR value up to 4 with an-ellipticity parameter is varying from 0 to 0.5 . Testing of synthetic data for single layer on vertical transverse isotropy (VTI) medium obtained the maximum residual error value produced by the Padé approximation is $0.25 \%$ in ODR $=4$. Therefore, Padé approximation is better than other methods for reducing residual moveout.
\end{abstract}

Keywords: Residual Moveout; Long Offset; Padé Approximation; Anisotop Medium

\section{Introduction}

Technology in oil and gas sector in Indonesia is not good enough. Whereas oil reserves are running low. Some of the factors needed to find new oil reserves are to add information from seismic data obtained. Processing and interpretation of seismic data usually assume a homogeneous isotropy medium. However, the real subsurface are generally heterogeneous anisotropy medium. Anisotropic properties of rocks can be seen at the Normal Moveout (NMO) correction. The aim of NMO correction to eliminate the effect of distance. The NMO correction depends on offset and velocity. The problem caused when Normal Moveout (NMO) correction is the stretching effect. The trigger for stretching effect is influenced by frequency attenuation of seismic waves experienced by incoming ray velocity received at a far offset [1].

The effect caused when seismic data acquisition was done at a long offset is non-hyperbolic moveout [2]. The non-hyperbolic that appears is a hockey stick caused by anisotropic factors that occur in subsurface of the earth so that it cannot be reduced by NMO correction with hyperbolic travel time equation. The inaccurate selection of normal moveout (NMO) velocity will cause a normal moveout residual. This problem can be solved with the muting of data in the processing, but it can affect the loss of information in subsurface such as lithology, fluid, etc [1]. Non-hyperbolic travel time equation is needed to generate the best acuration of NMO velocity and residual move out, so the processed of muting data can be minimized.

Some researchers have modified non-hyperbolic travel time equation. The purpose of modification is obtaining normal moveout (NMO) velocity more accurate so that the residual moveout value is smaller mainly in large offset to depth ratio (ODR). Hyperbolic traveltime equation was formulated by Taner and Koehler, but this equation is only accurate in offset to depth ratio (ODR) $\leq 1$ [3]. Travel time equation was also modified by Alkhalifah, which derived from travel time equation with an-ellipticity parameter $(\eta)$. However, this equation in offset to depth ratio $(\mathrm{ODR})<2$ [6]. Fomel and Stovas have also modified the equation, unfortunately, the accurately in offset to depth ratio (ODR) $\leq 2$ [4]. Three years ago, Song et al. was modified the equation the accurate of this equation in normalized offset $<2$ or offset to depth ratio $(\mathrm{ODR}) \approx 4.5[5]$. They suggest [4/3] and [7/6] order Padé approximation to applied for VTI media. The result obtained is the [7/6] order is better than [4/3] order Padé approximation.

In this study, will use the modified travel time equation using Padé approximation in orde [7/6]. The approximation will be compared with hyperbolic, nonhyperbolic method by Alkhalifah, and Fomel and Stovas method to see the best accuration, which indicates the smallest residual reduction. The best method of the travel time with the smallest residual can be applied for increasing the image quality.

\footnotetext{
* Corresponding author: syamsu.rosid@ui.ac.id
} 


\section{Theory}

Some researcher has proposed method for estimating travel time. The hyperbolic method used for isotropic medium. The travel time equation of hyperbolic method until second part of the Taylor series. The equation $(t)$ can be written as,

$$
t^{2}=t_{0}^{2}+\frac{x^{2}}{v_{N M O}^{2}}
$$

where :

$$
\begin{array}{ll}
x & =\text { offset }(\mathrm{m}) \\
t_{0} & =\text { travel time at zero offset }(\mathrm{s}) \\
v_{N M O} & =\text { velocity of } \mathrm{NMO}(\mathrm{m} / \mathrm{s})
\end{array}
$$

Hyperbolic method will cause the problem if applied in anisotropic medium on long offset and also can trigger other problem. The problem called hockey stick effect. Therefore, another better method is needed to solve these problems.

Quadratic part of travel time equation for anisotropic medium by Tsvankin and Thomsen have modified by Alkhalifah and Tsvankin [6]. The equation can be written as :

$$
\begin{aligned}
& t^{2}=t_{0}^{2}+\frac{x^{2}}{v_{N M O}^{2}} \\
& -\frac{2 \eta X^{4}}{v_{N M O}^{2}\left[t_{0}^{2} v_{N M O}^{2}+(1+2 \eta) X^{2}\right]}
\end{aligned}
$$

where :

$$
\begin{gathered}
v_{h}=v_{N M O} \sqrt{1+2 \eta} \\
\eta=0.5\left(\frac{v_{h}^{2}}{v_{N M O}^{2}}-1\right) \\
\eta=\frac{\varepsilon-\delta}{(1+2 \delta)} \\
v_{N M O}=v_{p 0} \sqrt{1+2 \delta}
\end{gathered}
$$

Fomel and Stovas have also modified hyperbolic travel time equation with generalized moveout approximation [4]. The equation of Fomel and Stovas involves five coefficients. The coefficients are used as comparison for two coefficient in hyperbolic functions. The coefficient can be written as :

$$
\begin{gathered}
t_{0}, v \\
A=-4 \eta, B=\frac{1+8 \eta+8 \eta^{2}}{1+2 \eta}, \\
C=\frac{1}{(1+2 \eta)^{2}}
\end{gathered}
$$

The equation of Fomel and Stovas method becomes [5]:

$$
\begin{aligned}
& t^{2}=t_{0}^{2}+\frac{x^{2}}{v^{2}} \\
& +\frac{A X^{4}}{v^{4}\left(t_{0}^{2}+B \frac{x^{2}}{v^{2}}+\sqrt{t_{0}^{4}}+2 B t_{0}^{2} \frac{x^{2}}{v^{2}}+C \frac{x^{4}}{v^{4}}\right)}
\end{aligned}
$$

Song, et al. have modified the travel time equation using Padé approximation [5]. The Padé approximation is a particular and classic type of rational function. This approximation has the idea of extending a function as a ratio of two power series and determine the coefficient of numerator and denominator using expansion of Taylor series coefficient of the function $f(x)$ [7].

An-elliptical parameter analysis using Padé approximation is done by deriving the travel time quadratic equation non-hyperbolic NMO velocity of quasi-P waves on vertical transverse isotropic (VTI ) medium. The numerical experiment has been carried out by Song, et al. to determining the best order obtained with a small residual of travel time [5]. Based on the numerical experiment by Song, et al. suggest for used order [4/3] and $[7 / 6]$, however the best accuracy obtained in order $[7 / 6]$.

\section{Methodology}

The method used in this study is to conduct forward modeling of the subsurface layering structure Forward modeling to obtain synthetic data that will be used as the main data to be processed and interpretation. Processed of forwarding modeling used the MATLAB program. The synthetic data will be processed and interpretation using the modified travel time equation using Padé approximation.
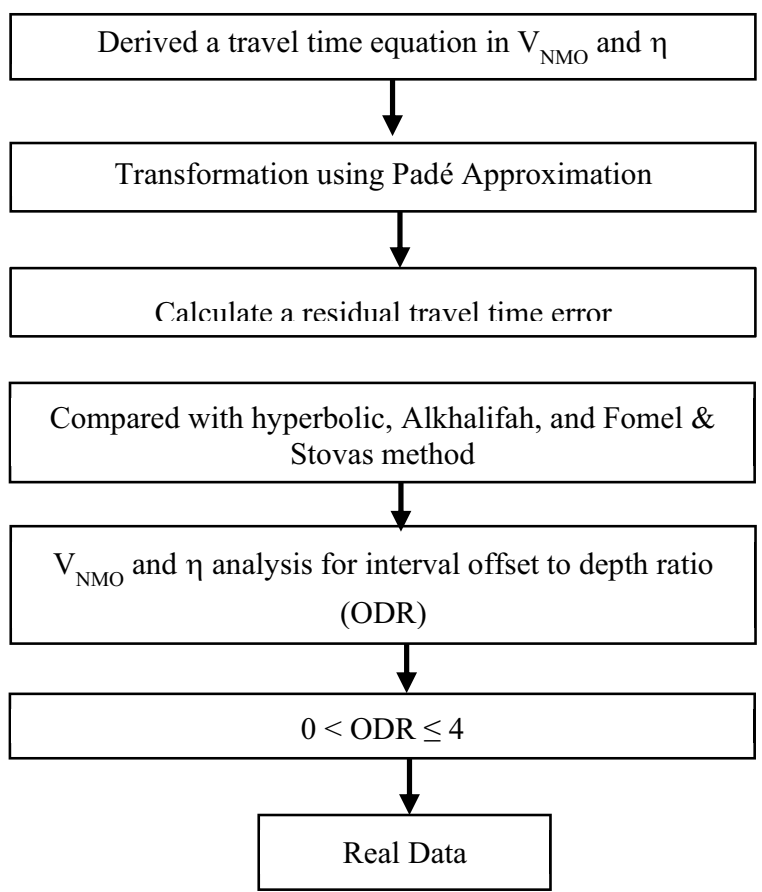

Fig. 1. Flowchart of Research

The steps of work are as follows:

a. Derive the hyperbolic travel time equation to non-hyperbolic travel time equation using Padé approximation to determine the residual error.

- Travel time polynomial by Taylor series $t^{2}=a_{0}+a_{1} x^{2}+a_{2} x^{4}+a_{3} x^{6}+\ldots$ 
- Transformation the polynomial using Padé approximation

The Padé approximation is a function of rational based on the expansion of power series in accordance with prescribed power series that is determined as high as possible [8-10]. The function of power series can be written in equation (8).

$$
f(\lambda)=\sum_{i=0}^{\infty} a_{i} \lambda^{i}
$$

The function of rational is

$$
R_{N M}(\lambda)=\frac{A_{N}(\lambda)}{B_{M}(\lambda)}=\frac{\sum_{k=0}^{L} A_{i} \lambda^{i}}{\sum_{i=0}^{M} B_{i} \lambda^{i}}
$$

defined as the Padé approximation if

$$
f(\lambda)-R_{N M}(\lambda)=O\left(\lambda^{N+M+1}\right)
$$

To solve the system, we used the normalization condition $B_{0}=1$. So that the approximation is the Maclaurin expansion for $f(\lambda)$. The coefficient of $A_{N}$ $(\lambda)$ and $B_{M}(\lambda)$ can be written in equation (11).

$$
\left\{\begin{array}{c}
A_{N}(\lambda)=A_{0}+A_{1} \lambda+\ldots+A_{L} \lambda^{N}, \\
B_{M}(\lambda)=1+B_{1} \lambda+\ldots+B_{M} \lambda^{M}
\end{array}\right.
$$

for requirements

$$
\begin{aligned}
& f(0)=R_{N M}(0) ; \\
& \left.\left.\frac{d^{i}}{d \lambda^{i}} f(\lambda)\right|_{\lambda=0} \frac{d^{i}}{d \lambda^{i}} R_{N M}(\lambda)\right|_{\lambda=0,} \\
& i=1,2,3, \ldots, N+M
\end{aligned}
$$

Multiply the denominator of $R_{N M}(\lambda)$ with equation 10 for get the coefficient of $A_{i}(\mathrm{i}=0, \ldots, \mathrm{N})$ and $B_{i}(\mathrm{i}=$ $1, \ldots \mathrm{M})$ and coefficient of $\lambda^{i}$ calculated by $\mathrm{i}=0, \ldots, N+M$. the result is

$$
\left\{\begin{array}{c}
\sum_{i=0}^{M} B_{k} a_{i-k}=A_{i}, i=0, \ldots, N, \\
\sum_{i=1}^{M} B_{k} a_{i-k}=0, i=N+1, \ldots, N+M
\end{array}\right.
$$

$a_{i}$ is coefficient of $\mathrm{i}$ in the Taylor series, shown in Appendix X. So that the equation 14 can be written as :

$$
\left\{\begin{array}{c}
a_{N}+a_{N} B_{1}+\cdots+a_{N-M+1} B_{M}=0 \\
\vdots \quad \vdots \\
a_{N+M}+a_{N+M-1} B_{1}+\cdots+a_{N} B_{M}=0
\end{array}\right.
$$

since $B_{0}=1$, equation (14) become linier equation with the unkwown coefficient of denominator, so obtained :

$$
\left[\begin{array}{ccc}
a_{N-M+1} & \cdots & a_{N} \\
\cdots & \ddots & \cdots \\
a_{N} & \cdots & a_{N+M-1}
\end{array}\right]\left[\begin{array}{c}
B_{M} \\
\vdots \\
B_{1}
\end{array}\right]=-\left[\begin{array}{c}
a_{L+1} \\
\vdots \\
a_{L}
\end{array}\right]
$$

From equation 15, coefficient of denominator may be found. The coefficient of numerator $A_{i}(\mathrm{i}=0, \ldots, \mathrm{N})$ follow the equation 10 by equating the coefficient of 1 , $\lambda, \cdots, \lambda^{N}$ can write in equation (16).

$$
\left\{\begin{array}{c}
a_{0}=A_{0} \\
a_{1}+a_{0} B_{1}=A_{1} \\
a_{2}+a_{1} B_{1}+a_{0} B_{2}=A_{2} \\
\vdots \quad \vdots \quad \vdots \\
a_{N}+a_{N-1} B_{1}+\cdots+a_{N-M} B_{M}=A_{N}
\end{array}\right.
$$

For normalized offset and travel time the coefficient of $a_{0}$ is always equal to 1 [6], therefore $a_{0}=1$, where $\lambda \equiv x^{2}$, coefficient of $A_{i}$ and $B_{i}$ can be solved for the Taylor series

$$
t^{2}=a_{0}+a_{1} x^{2}+a_{2} x^{4}+a_{3} x^{6}+\ldots \text { and }[\mathrm{N} / \mathrm{M}]
$$
order of Padé approximation can be written in equation (17).

$$
\tau_{N M}{ }^{2}=R_{N M}(\lambda)=\frac{1+\sum_{i=1}^{N} A_{i} x^{2 i}}{1+\sum_{i=1}^{M} B_{i} x^{2 i}}
$$

- Travel time polynomial by Padé approximation

$$
\tau_{N M}{ }^{2}=R_{N M}(\lambda)=\frac{1+\sum_{i=1}^{N} A_{i} x^{2 i}}{1+\sum_{i=1}^{M} B_{i} x^{2 i}}
$$

b. Forward modeling for single layer using synthetic data. The exact model that will be used is modeling of raytracing anisotropic.

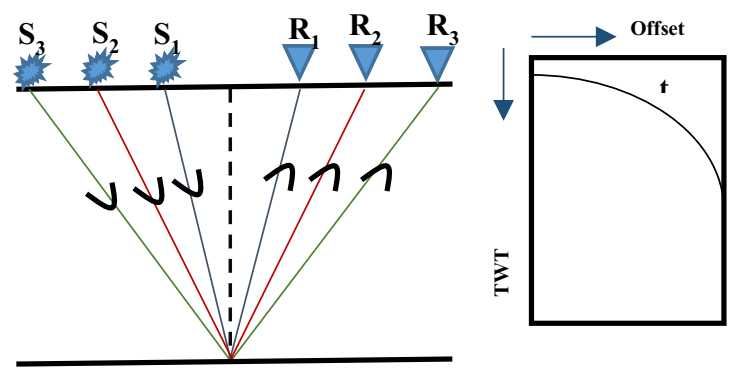

Fig. 2. Model of raytracing anisotropy

c. Calculate the residual travel time error of hyperbolic and non-hyperbolic equation with anisotropic parameter.

d. Compare a single layer residual travel time error of Padé approximation with other methods (hyperbolic, Alkhalifah and Fomel \&Stovas method).

e. Applied in real data (Wang, 2002). 


\section{Result and Discussion}

\subsection{Synthetic Data Analysis}

Single-layer research has been carried out on VTI medium using synthetic data. The data used to find residual error as follows :

- Depth $(\mathrm{h})=1000$ meter

- Offset $(\mathrm{x})=4000$ meter

The exact model used is based on anisotropy ray tracing approximation [11]. This research for compares an exact model of two-way time with another method such as hyperbolic, Alkhalifah, Fomel and Stovas and Padé approximation. The best method can be seen from the accuracy of the travel time value generated by the method that has the same value or close to the exact travel time.

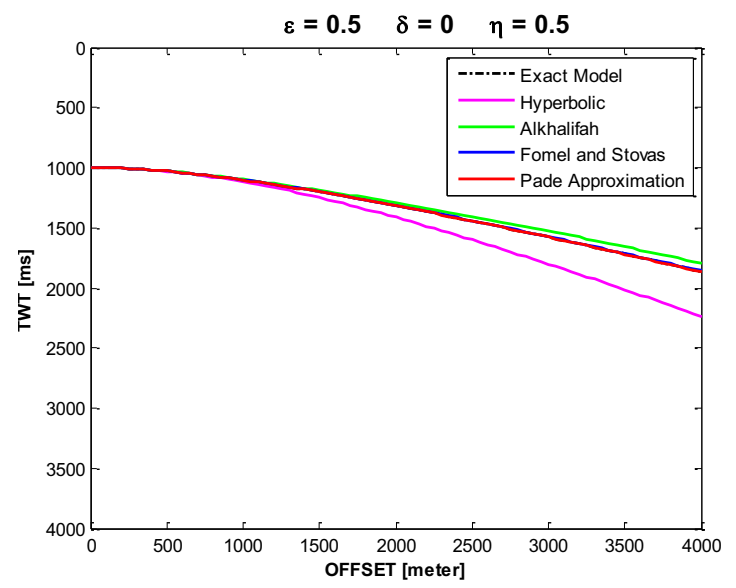

Fig. 3. The graph of Two Way Time [ms] vs Offset [m] between Exact model, Hyperbolic, Alkhalifah, Fomel and Stovas, and Padé approximation.

Figure 3 shows the graph of two-way time with an offset between exact model and comparison with all method. In this figure can be seen that the method has a travel time value that is close to the exact models is Padé approximation, whereas the Fomel and Stovas have little difference with the exact model with Alkhalifah and hyperbolic methods have travel time far from the exact models.

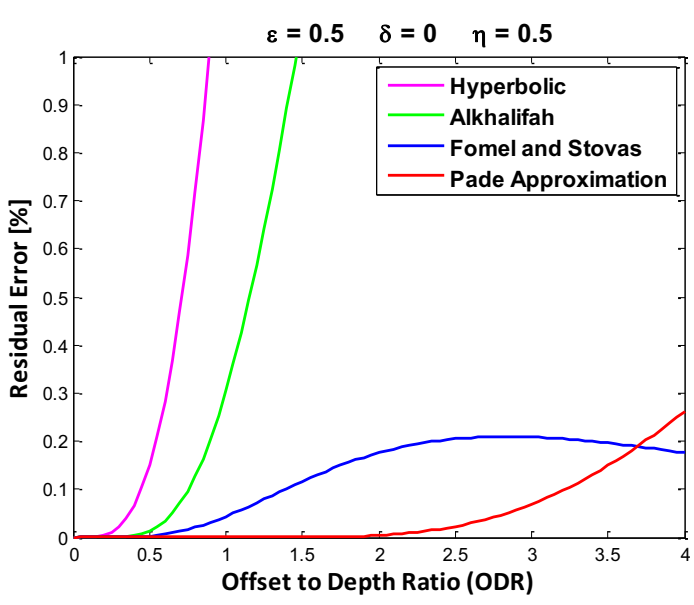

Fig. 4. Comparison residual error between hyperbolic, Alkhalifah, Fomel and Stovas and Padé approximation when $\eta$

$=0.5$ and offset to depth ratio (ODR) $\leq 4$.

Figure 4 shows the comparison residual error and offset to depth ratio between all method using shyntetic data with $\varepsilon=0.5, \delta=0$ and $\eta=0.5$. In this figure can be seen that some method have a big errors, especially for the hyperbolic method. Fomel and Stovas and Padé approximation have a small error when offset to depth ratio (ODR) $\leq 4$. However, Fomel and Stovas have a big error when ODR $\geq 0.5$ or after the offset 500 meters. Maximum residual error obtained by Padé approximation is $0.25 \%$ when offset to depth ratio 4 or offset 4000 meters.

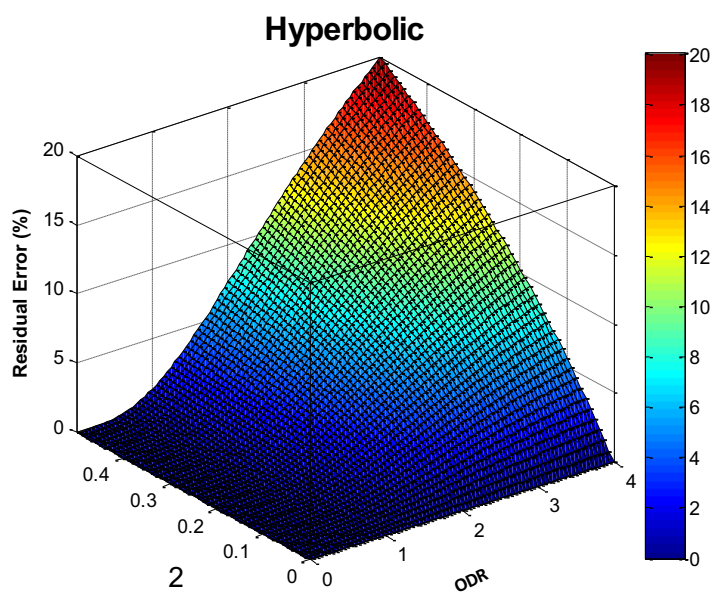

(a) 


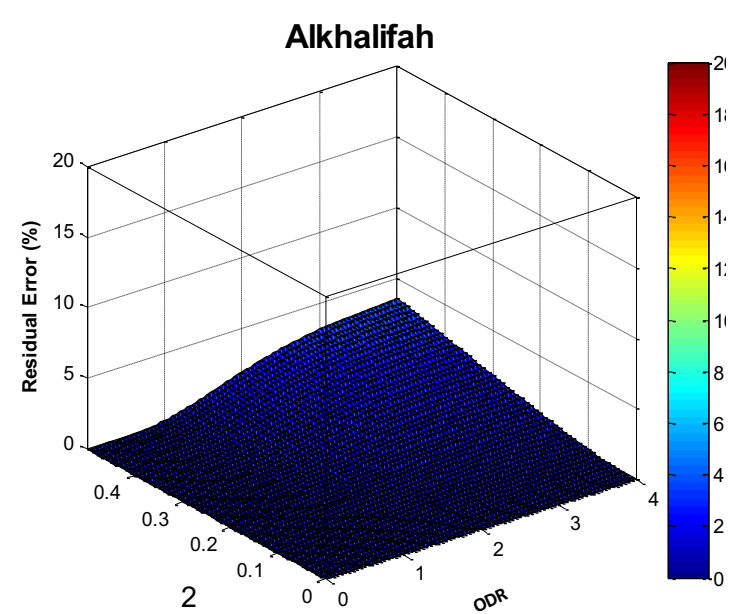

(b)

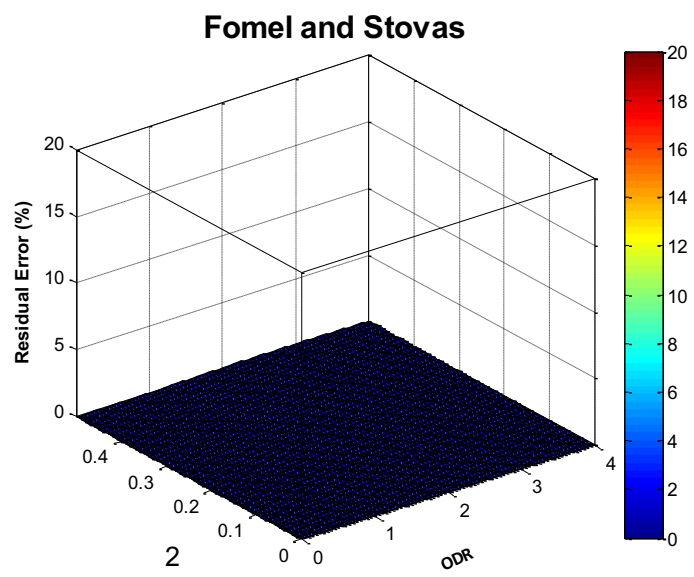

(c)

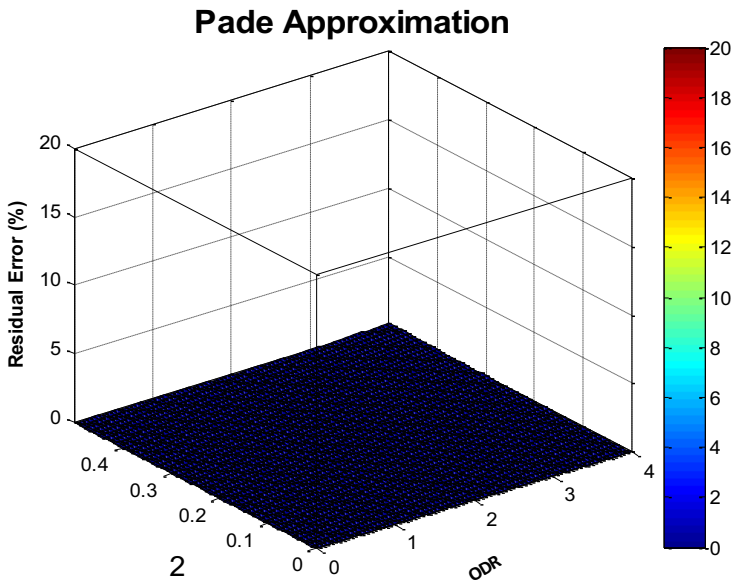

(d)

Fig. 5. 3D Plot of residual error between (a) hyperbolic, (b)Alkhalifah, (c) Fomel and Stovas (d) Padé approximation for varying etha from 0 until 0.5 with an interval 0.01 when Offset to Depth Ratio (ODR) $\leq 4$

Figure 5 shows 3D plots of residual errors between hyperbolic, Alkhalifah, Fomel Padé approximation. It shows the varying $\eta$ from 0 to 0.5 with an interval 0.01 when offset to depth ratio (ODR) $\leq 4$. When the anellipticity parameter $\eta$ is close to zero, all travel time method have an accurate enough. However, when $\eta$ is far from zero, most method like Alkhalifah and hyperbolic method listed high residual errors.

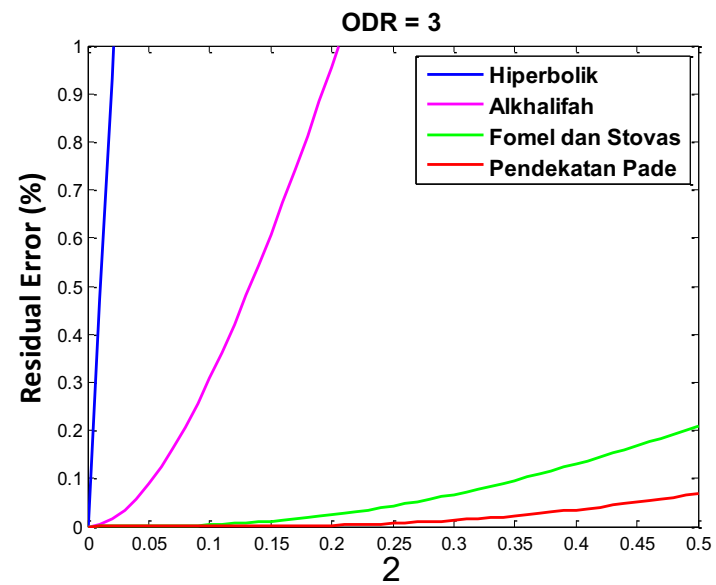

Fig. 6. The graph between residual error vs etha for hyperbolic, Alkhalifah, Fomel and Stovas and Padé approximation when $\mathrm{ODR}=3$

In Figure 6 shows that when offset to depth ratio 3, with varying etha comparison between Padé approximation was compare with Fomel and Stovas method shows that Padé approximation have a small residual error than Fomel and Stovas method, therefore Padé approximation has the best accurary for reducing residual error for increasing the image quality in seismic gather.

\subsection{Real Data}

The Real data used is laboratory test data [12]. For this research, we take three samples of data, which are Brinesaturated North Sea Shales, Brine-saturated Tight Sand and Brine-saturated Canadian Carbonates as shown in table 1 .

Table 1. The value of the sample laboratory test data [12]

\begin{tabular}{|c|c|c|c|c|}
\hline Sample & $\begin{array}{c}\text { Depth } \\
(\mathbf{m})\end{array}$ & Epsilon & Delta & Etha \\
\hline $\begin{array}{c}\text { North Sea } \\
\text { Shales }\end{array}$ & 1950.7 & 0.189 & -0.026 & 0.227 \\
\hline Tight Sand & 2483.6 & 0.009 & -0.074 & 0.097 \\
\hline $\begin{array}{c}\text { Canadian } \\
\text { Carbonates }\end{array}$ & 3588.8 & 0.023 & -0.101 & 0.155 \\
\hline
\end{tabular}




\subsubsection{Brine-saturated North Sea Shales}

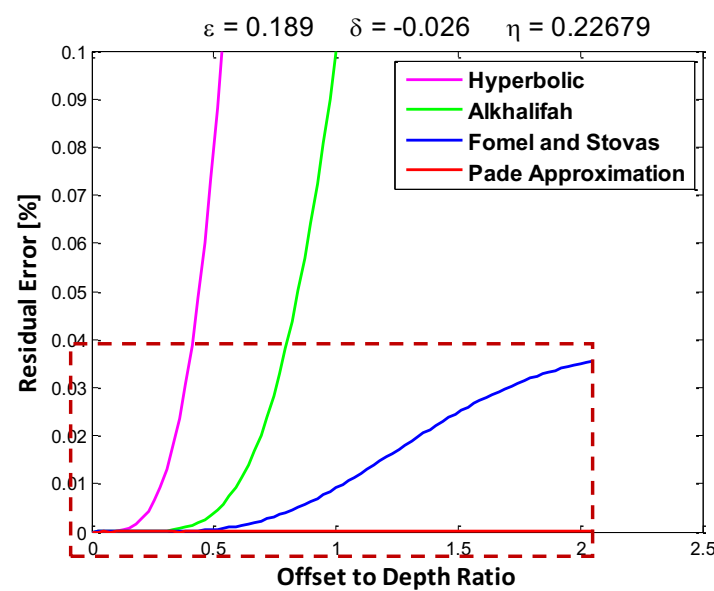

Fig. 7. Comparison residual error of several methods for Brinesaturated North Sea Shale

\subsubsection{Brine-saturated Tight Sand}

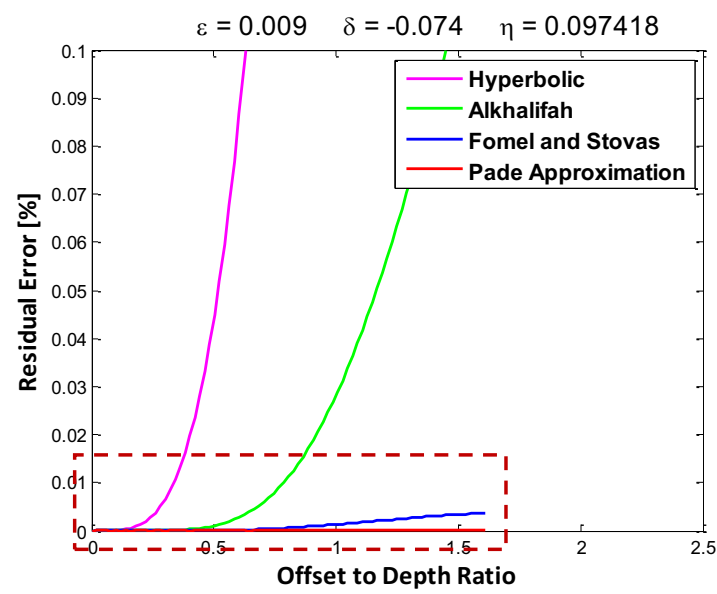

Fig. 8. Comparison residual error of several methods for Brinesaturated Tight Sand

\subsubsection{Brine-saturated Canadian Carbonates}

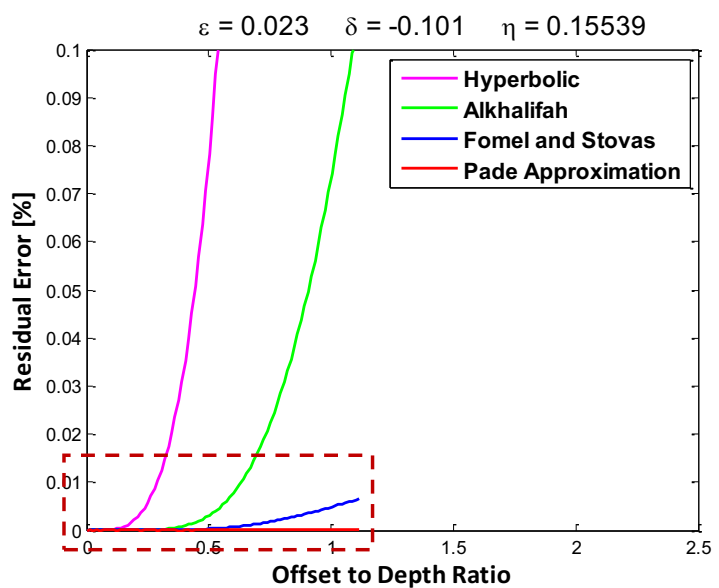

Fig. 9. Comparison residual error of several methods for Brinesaturated Canadian Carbonates
Table 2. The residual error for sample laboratory test data

\begin{tabular}{|c|c|c|c|c|c|}
\hline \multirow[b]{2}{*}{ } & \multirow{2}{*}{ 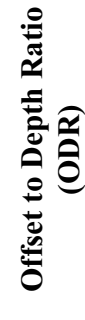 } & \multicolumn{4}{|c|}{ Residual Error (\%) } \\
\hline & & 苟 & 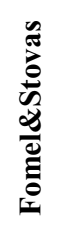 & 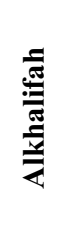 & 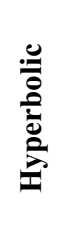 \\
\hline Shale & 2.05 & $\begin{array}{c}2.5 \mathrm{x} \\
10^{-4}\end{array}$ & $\begin{array}{l}3.5 \mathrm{x} \\
10^{-2}\end{array}$ & 0.7 & 4.7 \\
\hline Sand & 1.61 & $\begin{array}{c}3.5 \mathrm{x} \\
10^{-7}\end{array}$ & $\begin{array}{l}3.7 \mathrm{x} \\
10^{-3}\end{array}$ & 0.1 & 1.5 \\
\hline $\begin{array}{c}\text { Car- } \\
\text { bonates }\end{array}$ & 1.11 & $\begin{array}{c}3.6 \mathrm{x} \\
10^{-8}\end{array}$ & $\begin{array}{l}6.5 \mathrm{x} \\
10^{-3}\end{array}$ & 0.1 & 1.0 \\
\hline
\end{tabular}

Table 2 as shows the comparison of residual error for three sample laboratory test data, such as Brine-saturated North Sea Shales, Brine-saturated Tight Sand, and Brine-saturated Canadian Carbonates. From the table, seems like Padé approximation has smallest residual error than other methods for all samples. The minimum residual error of Padé approximation is $3.6 \times 10^{-8}$ on Canadian carbonates. Therefore, Padé approximation is the best method for reducing residual moveout for long offset data in VTI medium for increasing the quality of image in seismic section and the muting of seismic data can be minimized so that we will get a lot of subsurface information to find new oil reserves.

\section{Conclusion}

Padé approximation has the best accuracy in synthetic data, with smallest residual error for the an-ellipticity parameter $\eta=0.5$ when offset to depth ratio (ODR) $\leq$ 4. Residual error based on real data [12] for three samples can be seen that Padé approximation has smallest residual error than other methods for all samples. The minimum residual error of Padé approximation is $3.6 \times 10^{-8}$ in Canadian carbonates. Therefore, Padé approximation can be applied for increasing the quality of image in seismic section and the muting of seismic data can be minimized. 


\section{Appendix X}

\section{Taylor Series Coefficient}

$$
\begin{aligned}
& a_{0}=1 \\
& a_{1}=1 \\
& a_{2}=-2 \eta \\
& a_{3}=2 \eta(1+6 \eta) \\
& a_{4}=-2 \eta\left(1+16 \eta+52 \eta^{2}\right) \\
& a_{5}=2 \eta\left(1+30 \eta+240 \eta^{2}+544 \eta^{3}\right) \\
& a_{6}=-2 \eta\left(1+48 \eta+684 \eta^{2}+3648 \eta^{3}+6384 \eta^{4}\right) \\
& a_{7}=2 \eta\left(\begin{array}{l}
1+70 \eta+1540 \eta^{2}+14168 \eta^{3} \\
+56672 \eta^{4}+80960 \eta^{5}
\end{array}\right) \\
& a_{8}=-2 \eta\left(\begin{array}{l}
1+96 \eta+3000 \eta^{2}+41600 \eta^{3} \\
+280800 \eta^{4}+898560 \eta^{5}+1085760 \eta^{6}
\end{array}\right) \\
& a_{9}=2 \eta\left(\begin{array}{l}
1+126 \eta+5292 \eta^{2}+102312 \eta^{3}+1023120 \eta^{4} \\
+5437152 \eta^{5}+14499072 \eta^{6}+15189504 \eta^{7}
\end{array}\right) \\
& a_{10}=-2 \eta\left(\begin{array}{l}
1+160 \eta+868 \eta^{2}+222208 \eta^{3} \\
+3055360 \eta^{4}+23744512 \eta^{5}+103882240 \eta^{6} \\
+237445120 \eta^{7}+219636736 \eta^{8}
\end{array}\right) \\
& a_{11}=2 \eta\left(\begin{array}{l}
1+198 \eta+13464 \eta^{2}+439824 \eta^{3}+7916832 \eta^{4} \\
+83692224 \eta^{5}+530050752 \eta^{6} \\
+1968759936 \eta^{7}+3937519872 \eta^{8} \\
+3261380096 \eta^{9}
\end{array}\right) \\
& a_{12}=-2 \eta\left(\begin{array}{l}
1+240 \eta+19980 \eta^{2}+809856 \eta^{3} \\
+18424224 \eta^{4}+252675072 \eta^{5} \\
+2158266240 \eta^{6}+11510753280 \eta^{7} \\
+37122179328 \eta^{8}+65994985472 \eta^{9} \\
+49496239104 \eta^{10}
\end{array}\right) \\
& a_{13}=2 \eta\left(\begin{array}{l}
1+286 \eta+28600 \eta^{2}+1407120 \eta^{3} \\
+39399360 \eta^{4}+677668992 \eta^{5} \\
+7454358912 \eta^{6}+53245420800 \eta^{7} \\
+244928935680 \eta^{8}+697676362240 \eta^{9} \\
+1116282179584 \eta^{10}+765004570624 \eta^{11}
\end{array}\right)
\end{aligned}
$$

$$
a_{14}=-2 \eta\left(\begin{array}{l}
1+336 \eta++39732 \eta^{2}+2330944 \eta^{3} \\
+78669360 \eta^{4}+1654304256 \eta^{5} \\
+22677754176 \eta^{6}+207339466752 \eta^{7} \\
+1269954233856 \eta^{8}+5131128217600 \eta^{9} \\
+13084376954880 \eta^{10}+19031821025280 \eta^{11} \\
+12008172789760 \eta^{12}
\end{array}\right)
$$

\section{References}

1. Öz. Yilmaz. Seismic Data Analysis: Processing, Inversion, and Interpretation of Seismic Data, SEG Tulsa. (2001)

2. T. Guntoro, B.J. Santosa. Pre Stack Depth Migration Vertical Transverse Isotropy (Psdm VTI) Pada Data Seismik Laut 2D. Jurnal Sains Dan Seni Pomits, 3 : 2337-3520. (2014)

3. M.T. Taner, F. Koehler, Velocity Spectra-digital Computer Derivation and Applications of Velocity functions. Geophysics, 34: 859-881. (1969)

4. S. Fomel, A. Stovas, Generalized Nonhyperbolic moveout approximation. Geophysics, 75, 2 U9-U18. (2010)

5. H. Song, Y. Gao, J. Zhang, Z. Yao, Long-offset moveout for VTI using Pade approximation. Geophysics, 81, C219-C227. (2016)

6. T. Alkhalifah and I. Tsvankin, Velocity analysis for transversely isotropic media. Geophysics, $60 \mathrm{pp}$ 1550-1566. (1995)

7. G. A. Baker Jr., P. R. Graves-Morris, Pade Approximants. Encyclopedia of Mathematics and its Applications, 13 and 14, ed. G.-C. Rota (AddisonWesley). (1981)

8. G.A. Baker Jr., Essential of Padé approximants in theoretical physics. Academic Press. (1975)

9. Z. H. Ma. Modern applied mathematics handbook (in Chinese). Tsinghua University Press. (2005)

10. A.I. Aptekarev, V. Buslaev, A. MartínezFinkelshtein, and S.P. Suetin Padé, approximants, continued fraction, and orthogonal polynomials, Russian Mathematical Surveys, 66: 1049-1131 . (2011)

11. P. F. Daley, E. S. Krebes, L. R. Lines, A comparison of exact, approximate, and linearized ray tracing methods in transversely isotropic media, CREWES Research Report, 22: 1-21. (2010).

12. Z. Wang, Seismic Anisotropy in Sedimentary Rocks, Part 2: Laboratory Data, Geophysics, 67: 14231440. (2002) 